\title{
A case study based approach to the integration of sustainable design analysis, performance and building information modelling
}

\author{
B. Ceranic ${ }^{1}$, M. Faulkner ${ }^{1}$, A. Dean ${ }^{1} \&$ D. Latham ${ }^{2}$ \\ ${ }^{1}$ University of Derby, $U K$ \\ ${ }^{2}$ Latham Architects, UK
}

\begin{abstract}
This paper presents a case study based research of both the method and technology for the integration of sustainable design analysis (SDA) and building information modelling (BIM) within smart built environments (SBE).

Level 3 BIM federation and integration challenges are recognised and improvements suggested, including issues with combining geometry and managing attribute data. The research defines SDA as rapid and quantifiable analysis of diverse sustainable alternatives and 'what if' scenarios posed by a design team and client during the early stages of the project, where the benefits of correct decisions can significantly exceed the actual investment required.

The SDA concept and BIM integration findings are explained through a convergence from conceptualisation to calculation stages, emphasising the importance of an iterative over a linear approach. The approach allowed for a multitude of "what if" scenarios to be analysed, leading to more informed sustainable solutions at the right stages of the project development, with a generally lower level of detail (LOD) and computational/modelling effort required. In addition, the final stage of Building Regulations Part L compliance calculations was reached with a lot greater level of certainty, in terms of its requirements.

Finally, a strategy for long term performance monitoring and evaluation of the building design in terms of its environmental sustainability is presented, via integration between BIM and SBE (Smart Built Environment) technologies.

Keywords: building information modelling, sustainable design analysis, smart built environments, integration.
\end{abstract}




\section{Introduction}

The UK Government has committed itself, through its Government Construction Strategy (2011), to a five year programme for construction sector modernisation, central to which is the implementation of information rich building information modelling (BIM) technologies, protocols and collaborative methods, aiming to improve efficiency and procurement processes within the industry. The key objective is reducing capital cost and the carbon emissions from the construction and operation of the built environment by $20 \%$ [1]. This has had a direct impact on the rate of uptake of BIM in construction industry, with the drive becoming even more intense now that the Department for Business, Innovation and Skills has unveiled the Government Strategic Plan for Level 3 BIM (February 2015).

However, despite great advances in the technical interoperability and collaborative aspects of BIM, its integration with sustainable design is lagging behind and professionals are perplexed by the number and complexity of software solutions on the market [2]. Furthermore, the concept of "smart built environment" in terms of its integration with BIM is quite new and mostly at the research and development stage.

Still, given that the current research reports on the solutions for the above interoperability issues as technically possible [3]; why should we do it, what benefits can it bring, can it be adopted and with time can it prove itself over the current approach?

The costs of design changes and their impact on the project costs are inversely proportionate. As the design progresses from its feasibility to tender stage (and finally to the construction), the potential of cost savings reduces. Hence, the cost benefit of changes in design is at its highest at the early stages of design, when coincidentally the cost of undertaken design effort is at its lowest. This fact is widely published and visually interpreted via the 'MacLeamy Curve' [4].

Herein lies the real benefit of fully integrating sustainable design analysis within BIM enabled design process [5].

\section{BIM integration with SDA and SBE}

As defined in [6], "sustainable design analysis could be referred to as rapid and quantifiable feedback on diverse sustainable alternatives and 'what if' questions posed by a design team and client during the early stages of the project". Its main purpose is to maximise environmental versus cost benefits of the project through informed choices at early stages of design, based on timely feedback on different aspects such as building materials, construction specifications, energy consumption and generation, $\mathrm{CO}_{2}$ emissions, water use and harvesting, waste and pollution management. Of course, there are other facets which are inherently linked and therefore considered, such as: functional (constructional, operational), human (safety and security, comfort health and wellbeing), socio-cultural (context, sense of place, aesthetics) and economical (profits, environmental cost impact, life cycle costing etc.) [7]. Importance of SDA integration within a BIM 
enabled design process is thus self-evident, in particular at feasibility stages of design. Currently, in most small to medium design practices SDA is understood as a "one off" exercise by specialist companies, commissioned only when the construction drawings are complete. It is governed by the Building Control approval deadlines with usually very little time left before construction is scheduled to commence. Thus it is often too late for any meaningful changes to take place. A shift in design management and recruitment strategy is required, so that we can have a rapid and frequent access to sustainable design analysis expertise within the office, in particular at early stages of design.

The next level would then naturally be integration with smart built environments (see Fig. 1), where SBE refers to environments with smart objects, such as sensors and actuators, that communicate with the BIM and SDA within an integrated system, sufficiently 'smart' to intelligently control building energy consumption, whilst providing a comfort and support for their users [8].

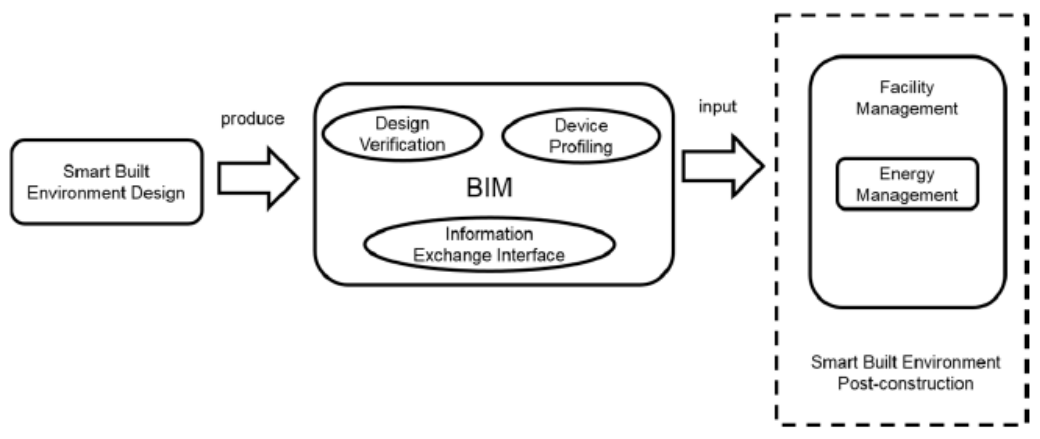

Figure 1: Concept of BIM and SBE integration [3].

The research defines the framework of SDA and BIM integration within the smart built environment through its convergence from conceptualisation to calculation stage, emphasising the importance of an iterative over a linear approach (see Figure 2). The integration is further reinforced and validated via building performance monitoring, real time data feedback, system optimisation and finally, technological solution for intelligent behaviour that minimises building energy consumption, provides comfort and support for users in their daily activities. Sustainable design analysis broadly follows two stages; conceptualisation and calculation [6]. They require diverse design approaches and serve different purposes for a distinct outcome. Conceptualisation stage challenges, interrogates and solves problems, trying to depict wider creative and rational constructs, the macro scale and the directional choices. The calculation stage is more analytical in its nature, setting out to quantify qualitative directional choices above and compare different sustainable design scenarios and alternatives.

For the latter stage there is plethora of environmental performance building programmes on the market, such as Integrated Environmental Solutions IES $<\mathrm{VE}>$, Autodesk Revit, Ecotect, Vasari, Green Building Studio, Graphisoft 
EcoDesigner STAR, EDSL, TAS Building Designer, EDR California, eQuest, U.S. Department of Energy, Energy Plus, Cymap Mechanical, DesignBuilder, etc. They all vary in terms of their capabilities and versatility of sustainable design, accuracy and data representation, the knowledge required to use, results interpretation, visual display and input/output type, building regulations compliance, sustainable codes assessment etc.

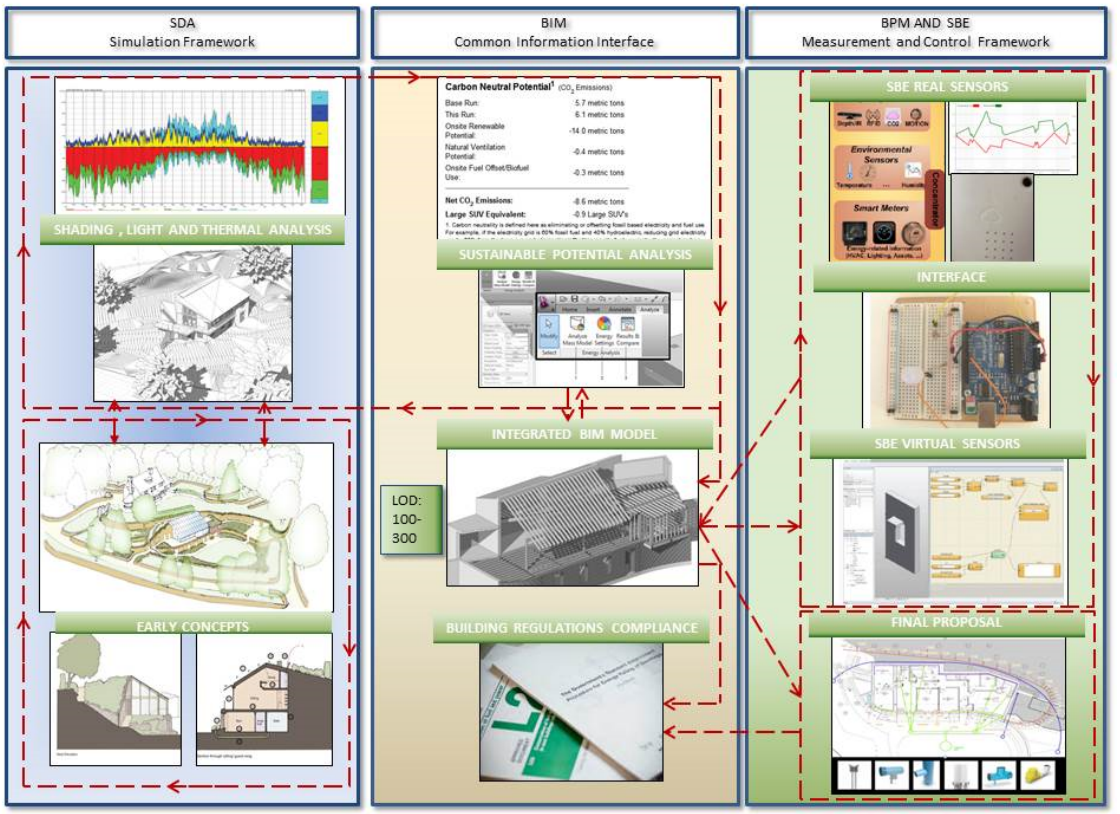

Figure 2: $\quad$ SDA BIM BPM SBE integration research approach.

\section{Research method}

The fundamental reason for choosing a case study approach was to investigate principles for detailed and complex analysis of framework for sustainable design and its integration with BIM and smart building environments through the prism of a complex and innovative real word project. Simons [9] summarises this view, stating that: "A case study is an in-depth exploration from multiple perspectives of the complexity and uniqueness of a particular project, policy, institution, programme or system in 'real life' context. It is research based, inclusive of different methods and is evidence-led. The primary purpose is to generate in depth understanding of a specific topic".

To ascertain a concise realisation of the research project a single-case study holistic design method has been chosen. Yin [10] states that the holistic design approach is beneficial when the theory pertinent to the case study itself is of a holistic nature or where no logical sub-units can be identified. However, he 
further asserts the importance of access to the real world detailed information on all stages of progress, stating that otherwise the case study may be carried out at an overly abstract level, with a lack of suitably clear measures or data.

\section{Case study}

Hieron's Wood is a four bedroomed dwelling, currently being built in the garden of an existing 1920s house, situated in a former quarry (see Fig. 3(a), (b)). The property is located on the edge of the sustainable settlement of Little Eaton, Derbyshire. The design concept was to produce an autonomous building related to its physical, historical and visual context, adopting its character through the selection and use of materials and imposing a very low imprint on the site. Initial energy design calculations estimate that the house will produce more energy from the renewables than it will import externally. The project represents a unique opportunity to undertake long term research in three key areas; a) integration of BIM and monitoring of building performance regarding energy consumption/embodied carbons/health and wellbeing; b) innovative use of sycamore/other materials and technologies; c) detail design and construction.

The proposed house is comprised of:

- A lower ground floor containing an ensuite master bedroom, two ensuite guest bedrooms and a bed/study room; lower entrance hall, utility and a garden store accessed externally (see Fig 4);

- An upper ground floor with an open living/dining/ kitchen area, larder, cloakroom/wc and entrance hall;

- A small mezzanine open study balcony incorporated within the upper living dining area;

- A wide stair links the upper and lower ground floors and a light, glass stair accesses the mezzanine.

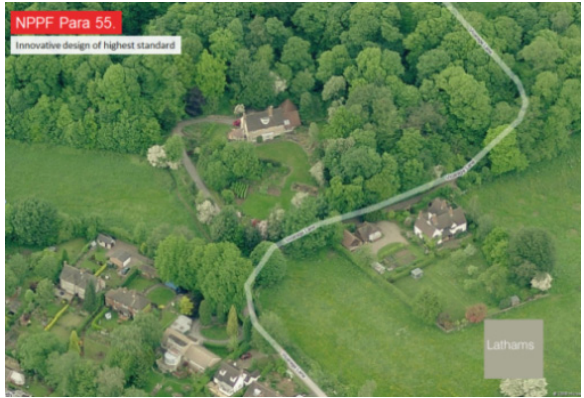

(a)

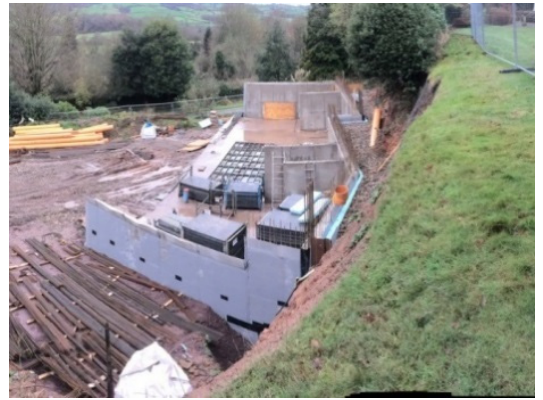

(b)

Figure 3: (a) Aerial view - site location; (b) Site build progress - March 2015.

The building exemplifies both proven and emerging green technologies alongside passive energy saving measures, such as a passive stack and earth tube natural ventilation. Design embodies the principles of 'fabric first' approach, 
through the creation of a highly insulated, breathable building, constructed using local materials, harnessing local resources and craft skills. Both sycamore and dry stone walling is sourced within the compounds of the site, situated in the former quarry. The proposed house takes full advantage of the site's sloping southerly aspect and reaching views across the valley, whilst harnessing the power of the sun to meet demand for electricity and hot water.

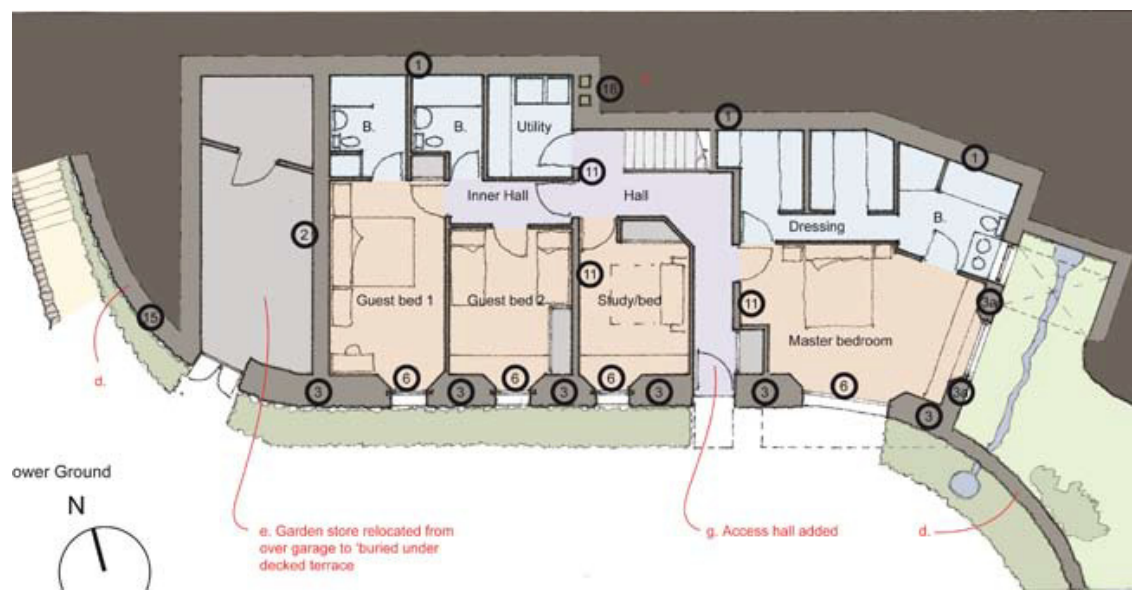

Figure 4: Lower ground floor.

Due to the unique nature of the project and special arrangements with Building Control detailed drawings are still being produced, although the works on the site have already commenced (June 2014 start, June 2016 completion). Hence, once the BIM model is finalised and a full drawings package and performance specification produced, the model will be exported to for final energy analysis and building regulations compliance calculations.

\subsection{BIM and sustainable design integration approach}

A conceptual BIM massing model was generated at the feasibility stage of design, based on early concept ideas and sketch schemes. The site sustainability potential was explored to define an optimal location, shape and building orientation, based on the site environmental context. For the first round of feedback an initial thermal study and energy consumption calculations were produced, based on a choice of limited number of predetermined building construction options. Thus, a number of feasible alternatives were rapidly created and their performance evaluated, without commitment to a "fixed" construction and services configuration so early in the design process.

The sustainable design analysis then proceeded to consider building energy consumption and costs, including water usage analysis, renewables and carbon neutrality potential (see Fig. 5). As the design progressed the building's thermal zones were defined and enclosures formed (walls layout, fenestration, roofs, floors and internal partitions). 


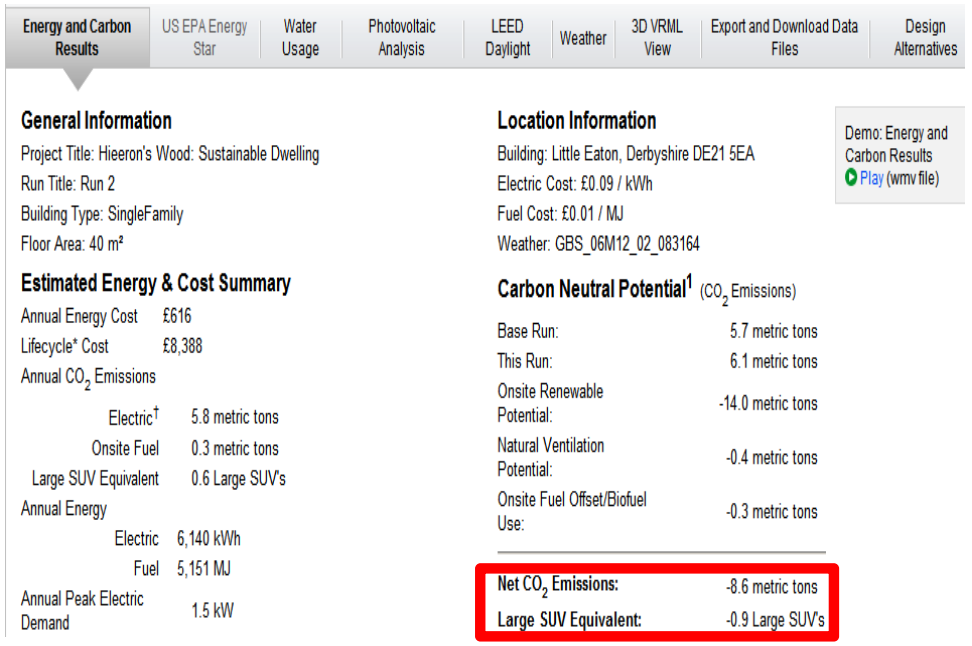

Figure 5: Carbon neutral potential analysis.

The model was then used for a final building shading and sun studies to determine sun path and solar stress diagrams, with room level calculations such as average daylight and artificial light factors, including visibility studies and solar exposure analysis [11] (see Fig. 6).

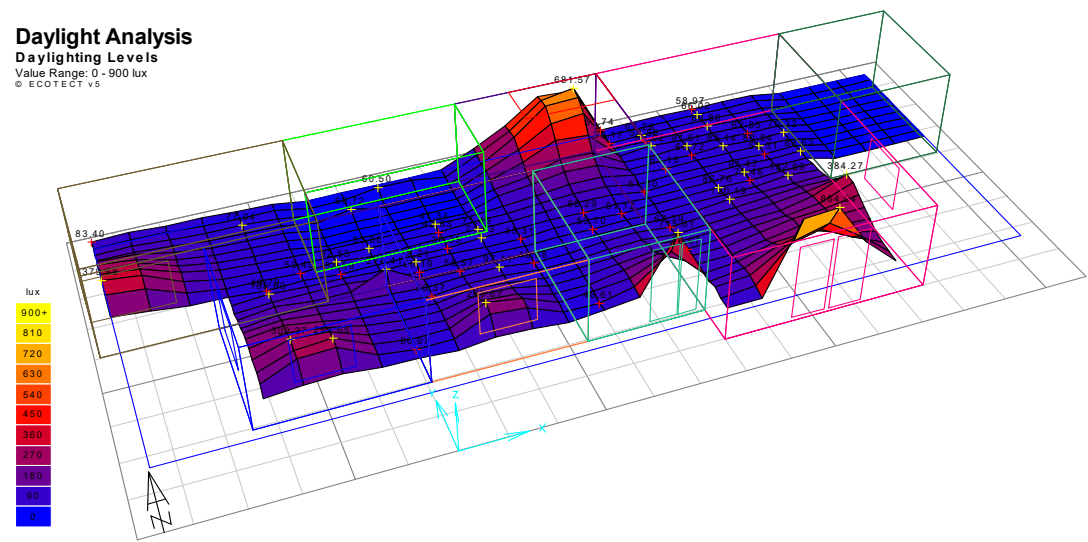

Average Value: 116.19 lux
Visible Nodes: 491

Figure 6: Overcast daylight analysis example.

Thermal templates for zones, services and occupancy patterns were defined at the final stage of analysis, generating thermal performance calculations. These included hourly temperature profiles, heating and cooling loads, passive gains 
and losses and incident solar radiation [12]. Based on those results zones were redesigned and the size, shape and position of apertures defined, including protection against excessive solar gains in the summer and a choice of materials and U-values (see Fig.7). It was at this stage that the design was 'fixed' and site work commenced.

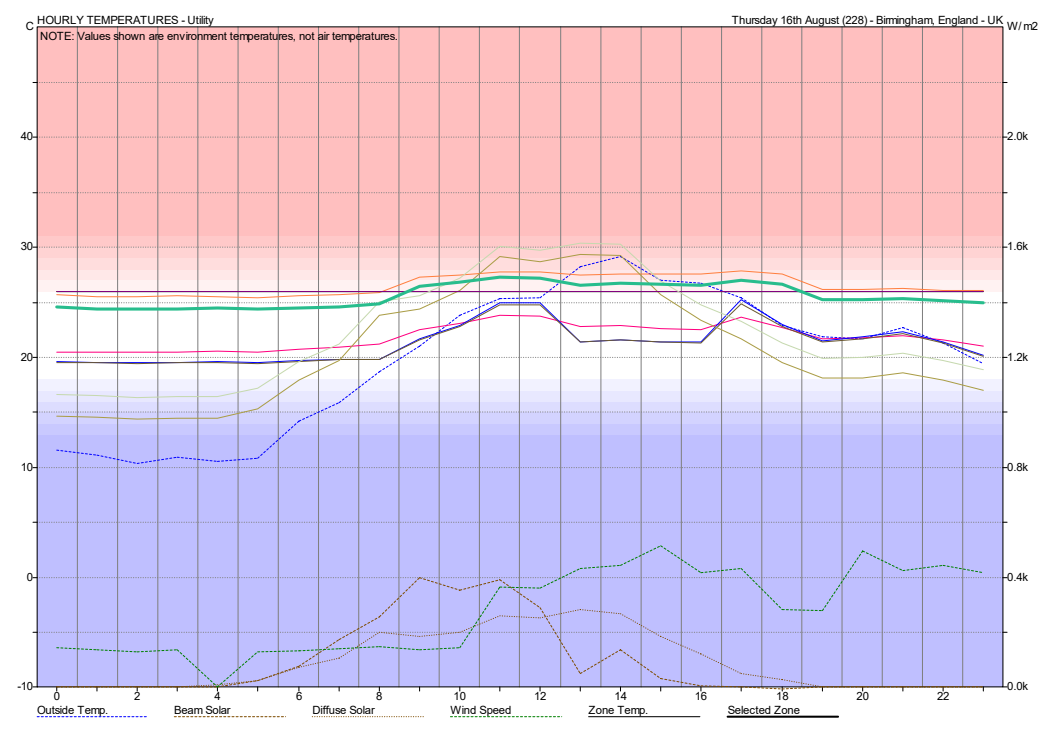

Figure 7: Example of hourly temperature profile - hottest day (average) August 16th.

\subsection{Building performance monitoring, BIM and SBE integration}

A long term performance monitoring (at least five years) of the Hieron's Wood development is proposed [13].

The performance strategy shown in Fig. 8 will include regular testing of the moisture content, condition and integrity of the sycamore structural frame, including temperature and relative humidity of its immediate surroundings. A digital micro probe (DmP) will be used to detect and analyse wood decay [14]. The monitoring parameters for earth tube system will include humidity, temperature, $\mathrm{CO}_{2}$ and VOCs in terms of air quality, but also air speed and the pressure [15]. Hempcrete performance monitoring will include temperature, relative humidity and water vapour transfer rate; but also heat flux sensors to monitor dynamic changes of U-value as it goes through its intermittent periods of wetting and drying. Thermal imaging analysis and air pressure testing will be conducted upon the completion of the build, with the former repeated at regular intervals thereafter. In addition the health of the occupants of the building will be monitored by specialist equipment recommended by Institut für Baubiologie + Nachhaltigkeit (IBN). 
Integrated BIM system will be extended from the design stage to provide interface for smart objects information exchange. For the post-construction phase, the prototype of BIM integrated facility management tool that provides advanced energy management of SBEs is proposed, with both smart objects and distributed energy resources (DERs) deployed [8].

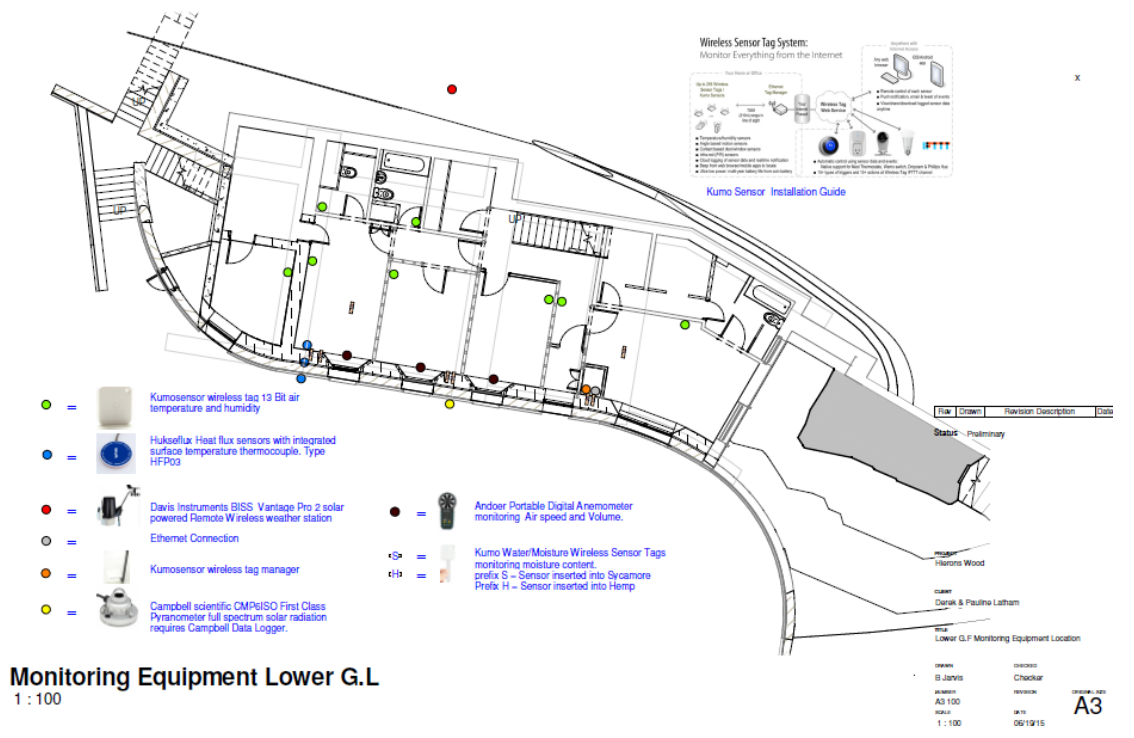

Figure 8: Building performance monitoring strategy - lower ground floor.

The first phase of sensor-actuator control is to profile smart objects within BIM environment (see Fig. 9), creating virtual sensors with IFC shared parameter field which defines type of the sensor. Hence, new BIM objects, for example smart windows (e.g. able to self-actuate to control their light transmission properties), can be generated and so optimised, controlling the real building component via its sensor-actuator connection $[8,16]$.
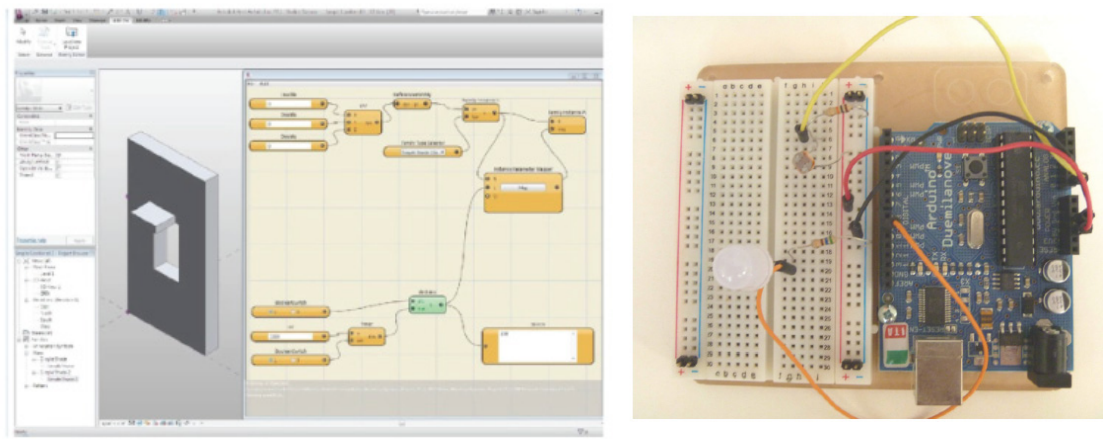

Figure 9: $\quad$ Prototype of BIM and real time data sensor integration in SBE [17]. 


\section{Discussion}

This case study based research presents the method and technology for integration of sustainable design analysis (SDA) with building information modelling (BIM) and smart built environments (SBE). The SDA validation via building performance monitoring and real time data feedback is currently being researched, together with system optimisation and technological solution for intelligent building energy consumption. During the feasibility stage of SDA an early building massing model was established and site sustainability potential investigated. On the building scale, orientation, shading, lighting, passive stack, natural ventilation and solar radiation analysis was conducted, together with thermal study and energy consumption calculations. These were initially conducted using a number of predetermined building construction specifications, thus generating energy calculations without committing to exact construction. Lastly the design was "fixed" and final analysis undertaken. The key findings are:

- Better design. Better understanding of the site, context, building passive features and services integration at early stages of design. Led to cc $40 \%$ improvement in the energy performance, from an initial to a final concept.

- Lower level of BIM development (LOD). LOD:100 to LOD:300 required, resulting in less computational/modelling effort needed. In addition, the final stage of Building Regulations Part L compliance calculations was reached with a lot greater level of certainty in terms of its requirements.

- Cost saving in design. At early stages of the project the 'trade of' between the cost of design changes and their impact on the overall project costs is at its most beneficial.

- BIM federation model and attribute data issues. A better solution for zone surface and volume recognition problems is required, together with an improvement to bi-directional links and backward compatibility between models. Hence the changes made in sustainable design analysis software should be directly reflected in the BIM. Furthermore, the performance degradation was noted with too many parameters attempting to be interoperable, thus affecting performance. Interpretation of BIM elements is overcomplicated by SDA software or plugin exports, leading to the loss of parametric intelligence. Finally, the software used in this research was rather US orientated, especially in relation to its sustainable assessment codes.

\section{Conclusions}

This case study and wider research concludes that despite the sustainable drivers, knowledge and understanding of SDA and BIM integration is still beyond smallto-medium practices and is not likely to be achieved in the near future. Furthermore, for it to be validated and lessons from mistakes learnt, it should be integrated within smart built environments (SBE) with a real time building performance data feeding back into the BIM system. This could not only help to 
analyse and optimise design decisions but also offer a framework for an intelligent life cycle building energy consumption control, providing a comfort and support for users in their daily activities.

Sustainable design analysis does help to produce better designs but the BIM integration process is not faultless or fully interoperable. With evolving technologies and frequent material advances databases can quickly become outdated and it is important that customisation options allow for full interoperability. BIM and SDA integration within smart built environments is still a relatively new concept and for its success case studies of this type are needed to test real word complex scenarios and uncover the problems that exist. Full consideration needs to be given to the global market and the difference in regional materials, construction methods, standards and regulations. Furthermore without careful controls and protocols being placed on the changes made in federated BIM model, the SDA work undertaken can soon become obsolete as BIM interoperability does not provide for automatic re-analyses.

Of course, the bespoke nature of Hieron's Wood case study does not solve the many questions required for "scaling up" and mass market construction solutions, but it does serve a useful purpose of learning from the lessons of "one off" innovative design case study. The building stemmed from a unique concept, brief and site conditions, with decisions made by a small team of practitioners and researches who on the outset agreed on producing an energy plus autonomous house using locally sourced materials, breathable envelope solutions and maximising passive design approaches. Restrictions imposed by tight budgets have been avoided and with no client in the true sense the flexibility of the design has been greater than on standard projects.

So, on the standard projects with tight deadlines and budgets, at which stages SDA should be integrated within the BIM design process and how much influence should it have? The answer remains same, it should be during those early design stages where it is ideally placed to maximise energy saving potential and overall sustainability performance. But in the real world this is rarely possible, as there is often no trained professionals or SDA software available within smaller architectural practices. On the other hand, clients are continuously challenging designers to improve energy consumption, looking to minimise its total costs throughout the building life cycle. Hence, for it to be really effective and lead seamlessly to the building regulations compliance stage, the shift in knowledge and design management is needed, together with an investment in recruitment and, ultimately, complete BIM integration. Thus, a sustainable design analysis expertise within practice is a key to the implementation process, not just consideration of BIM systems and their software integration.

\section{Acknowledgements}

Derek Latham - Architect and Home Owner, Price \& Myers - Structural Engineers, ARUP and Vaillant - Building Services Consultancy. 


\section{References}

[1] NBS, National BIM Report 2015, pp. 08-18, RIBA Enterprises Ltd, 2013

[2] Jrade A. and Jalaei F., Integrating building information modelling with sustainability to design building projects at the conceptual stage, Journal of Building Simulation, 6(4), pp. 429-444, 2013.

[3] Cormier, A.; Sylvain, R.; Pierrick, R.; Louis, S.; Etienne, W. Towards a BIM-Based Service Oriented Platform: Application to Building Energy Performance Simulation, Proc. of the 12th Conference of International Building Performance Simulation Association, Sydney, Australia, 2011.

[4] D. Light, BIM Implementation - HOK buildingSMART', 2011, online

[5] Bahar Y., Pere C., Landrieu J. and Nicolle C., A Thermal Simulation Tool for Building and Its Interoperability through the Building Information Modelling (BIM) Platform', Buildings 3(2), pp. 380-398, 2013.

[6] Ceranic B., Emmitt S., Architectural Technology - Research and Practice (Chapter 3), John Wiley \& Sons Inc., 2013.

[7] Krygiel, E. Nies, B. Green BIM: Successful Sustainable Design with Building Information Modelling, Indianapolis, Wiley Publishing Inc., 2008

[8] J. Zhang, B. Seet \& T.T. Lie, Building Information Modelling for Smart Built Environments, Buildings, 5, pp. 100-115, 2015.

[9] H. Simons, Case Study Research in Practice, pp. 21, Sage Publ. Ltd 2009

[10] Yin, R.K., Case Study Research: Design and Methods (Applied Social Research Methods), pp. 50, Sage Publications, Inc., 2009.

[11] Jalaei F. and Jrade A., An Automated BIM Model to Conceptually Design, Analyse, Simulate, and Assess Sustainable Building Projects, Journal of Construction Engineering, 2014.

[12] Schlueter A. and Thesseling F., Building information model based energy/exergy performance assessment in early design stages, Automation in Construction, 18(2), pp. 153-163, 2009.

[13] Tse P. and Colmer M., Building Performance Evaluation: Overview of Programme Portfolio, Innovate UK, 2014, online

[14] SIBTEC DmP, http://www.sibtec.com/digitalmicroprobe.html

[15] Lee, K. \& Strand, R., The cooling and heating potential of an earth tube system in buildings. Energy and Buildings, 40, pp. 486, 2008.

[16] Welle, B., John, H., Zack, R., ThermalOpt: A Methodology for automated BIM-based multidisciplinary thermal simulation for use in optimization environments, Building Simulation (4), pp. 293-313, 2011.

[17] K. Kensek, W. Kahn, Integration of Environmental Sensors with BIM Seven Case Studies, BESS - SB13 California: Advancing Towards Net Zero, pp. 29-35, 2013. 